\title{
GAME THEORETIC ANALYSIS OF IDEOLOGICALLY BIASED CLICKBAIT OR FAKE NEWS, AND REAL NEWS
}

\author{
KJELL HAUSKEN ${ }^{*}$ \\ Faculty of Science and Technology, University of Stavanger, 4036 Stavanger, Norway
}

\begin{abstract}
A decision and game theoretic model is developed for how one and two news organisations strike balances between producing clickbait or fake news, and real news. Each news organisation seeks to attract gullible consumers who consume more clickbait or fake news than real news, and non-gullible consumers who conscientiously consume only real news. Increasing a news organisation budget results in obtaining both more clickbait or fake news, and more real news. More clickbait or fake news is produced if the news organisation's unit cost of effort to produce real news, the production efficiency for clickbait or fake news, and the fraction of consumers consuming clickbait or fake news, increase. In contrast, less clickbait or fake news is produced if a news organisation's unit cost of effort to produce clickbait or fake news, and the production efficiency for real news, increase, and the gullible consumers consume real news with a higher frequency. Lower unit effort costs and higher budget and production efficiencies cause higher utility for a news organisation and lower utility for the competing news organisation. Higher weight assigned to the contest over clickbait or fake news induces both news organisations to exert higher effort to produce clickbait or fake news. When the gullible consumers of a news organisation consume a relatively large amount of real news in comparison to the consumers of another news organisation, then the first news organisation exerts higher effort to produce real news and obtains higher utility than the other news organisation.
\end{abstract}

Keywords: strategy, decision, news, fake news, real news, ideology, company, consumers

\section{Introduction}

\subsection{Background}

What clickbait, i.e., content designed to encourage consumers to click on a link to a web page and fake news have in common is that they may conflict with real truthful

*Email address: kjell.hausken@uis.no

Received 7 August 2019, accepted 5 July 2020 
news. News organisations face three challenges modelled in this article. The first is between producing clickbait or fake news, and real news. The second is to assess gullible consumers consuming a combination of news, and non-gullible consumers conscientiously seeking out and consuming only real news. The third is competition between news organisations, also facing the first two challenges.

The three challenges are impacted by each news organisation's potential ideological preference for what kind of clickbait or fake news to produce, as opposed to producing real and ideologically neutral news. A news organisation may be sponsored and controlled by one or several companies with ideological preferences. The control or influence may take various forms, such as decisions pertaining to hiring, firing, salaries, promotions, career opportunities, and political influence. These decisions may impact a news organisation positively if it is willing to produce clickbait or fake news in accordance with the interests of the ideologically biased company.

Potential ideological impact on news organisations may be influenced by changes in the news landscape. Over the last century examples of concentrated control over independent news media have emerged. For example, Lutz [8] reports that in 1983, 50 companies owned $90 \%$ of the American media. However, in 2011, only six companies, i.e., General Electric, News Corp, Disney, Viacom, Time Warner, and CBS, owned the same $90 \%$ of the American media.

Further impact on news organisations follows from the extent to which consumers consume news, which increases revenue from subscriptions and advertisers. Some advertisers may prefer consumption as such, regardless of whether the consumed news is clickbait or fake news, or real news. Other advertisers may prefer consumers of one or the other type.

The distinction between gullible and non-gullible consumers may tentatively be obtained by some kind of objective assessment, or assessment based on various criteria to be determined. While others may see consumers of fake news as gullible, fake news consumers may not see this as gullibility, but rather as loyalty to their group. One feature of contemporary mass media journalism seems to be a tendency or fixation on aligning with partisan 'narratives', i.e., what the entertainment industry usually terms 'tropes', i.e., fitting observed facts selectively to align with the narrative rather than with reality. Such narratives are usually crafted to align with various biases.

\subsection{Contribution}

The independent decision making process and the strategic interactions between news agencies in producing fake news have not been extensively studied. This paper fills this gap by first developing a decision model for one news organisation, and thereafter by studying a game theoretical model between two news organisation. The model is developed where each news organisation chooses which fraction of each budget to 
allocate into producing clickbait or fake news. The remaining fraction is allocated into producing real news. In making its decision, each news organisation accounts for which fraction of the consumers is gullible, in the sense of consuming clickbait or fake news more than real news, and which fraction is non-gullible, in the sense of consuming only real news. Each news organisation further accounts for competition with the other news organisation, which can be interpreted as the rest of the news industry, analogously determining which fractions of their budgets to allocate to clickbait or fake news, versus real news, while seeking to attract the same gullible and non-gullible consumers.

News organisations and consumers are in a symbiotic relationship. Ideological and other biases usually exist, on the one hand, within news organisations, their sponsoring and controlling company (or companies), owners, managers and personnel and, on the other hand, among consumers. How, when, and where the biases emerge can be hard to determine, and may be due to back and forth causation over time. On the one hand, the frequent and public altercations between US president Donald Trump and a number of major media owners (including $\mathrm{CNN}$ ) suggest biases within news organisations and those that control these. However, more generally, if a media organisation produces biased content that does not sell, then it will go bankrupt and cease to exist, unless heavily subsidised by its owner. Hence, on the other hand, bias in news organisations may be an adaptation to bias in the news consumer market. News organisations then staff their organisations and align their narratives to meet market demand. An example of this is the Macedonian fake news industry in 2016, catering to conservative audiences in the US and generating revenue from online advertising services [10]. The model in this article does not specify how, when, and where biases emerge, but assumes that biases may exist. The model then illustrates how news organisations and consumers interact, given their preferences and beliefs.

\subsection{Literature}

Regarding clickbait, Khoja [5] examines frequently shared articles, finding that $79 \%$ had an element of shock in the title, $67 \%$ contained an unknown or new concept, $63 \%$ also mentioned recent events in the news and media, $63 \%$ made a pop culture reference or mentioned food, $29 \%$ of the most shared titles mentioned 'you', 'I' or hinted at a personal story, $17 \%$ were listicles, and $8 \%$ mentioned an animal. Furthermore, $4 \%$ of the assessed titles contained only one of the seven factors, $8 \%$ of the titles two clickbait factors, $46 \%$ of titles three of the factors, and $42 \%$ four of the clickbait factors mentioned in this article. For further research on clickbait, see Blom and Hansen [2] and Zannettou et al. [12]. See also Kshetri and Voas [6] for some economic considerations of fake news, and Allcott and Gentzkow [1] regarding social media and fake news in the 2016 US election. See Hausken [4] for a game theoretic analysis of two media organizations exerting manipulation efforts to support their preferred actor among two 
actors interacting controversially. Tribe [11] illustrates implicit ideological biases in tools applied into policy science. Levins [7] and Nagy et al. [9] suggest that truth may be approximated by multiple independent models.

Article organisation. Sections 2 and 3 present models with one and two news organisations, respectively, with their analysis. Section 4 concludes the examination.

\section{One news organisation}

\subsection{The model}

Assume that news organisation $i, i=1, \ldots, n, n \geq 1$, has a budget $b_{i}$ that is converted into exerting effort $f_{i}$ to produce clickbait or fake news at unit cost $a_{i}$. Effort $f_{i}$ is news organisation $i$ 's one and only strategic choice variable. The remaining budget is used to exert effort $r_{i}$ into producing real news at unit $\operatorname{cost} d_{i}$, i.e.

$$
b_{i}=a_{i} f_{i}+d_{i} r_{i}
$$

The unit cost $a_{i}$ of producing clickbait or fake news can be higher, but it is usually lower than the unit cost $d_{i}$ of producing real news. It can also be significantly lower, expressed as $a_{i} \ll d_{i}$, as there is usually no requirement for validation of the news content. Much fake news is produced when journalists encounter an appealing and possibly untrue factoid that aligns with a narrative, and use it as a basis of a report without determining its veracity. Additionally, $a_{i}$ depends on news organisation $i$ 's value system, including which kinds of clickbait or fake news it is willing to produce. The budget may consist of monetary resources $M_{i}$ and non-monetary resources $N_{i}$ drawn from non-monetary valuation systems, i.e.

$$
b_{i}=M_{i}+N_{i}
$$

Distinguishing between monetary and non-monetary resources is akin to distinguishing between economic value, influence value (including symbolic, political, and economic prestige), and human value [3]. That is, news organisation $i$ first determines its monetary budget $M_{i}$, and thereafter its non-monetary budget $N_{i}$ which depends on its value system. News organisations vary greatly in their value systems which may cause, widely different budgets $b_{i}$ for organisations with the same monetary budget $M_{i}$. This, in turn, impacts the news organisations' ability and willingness to produce clickbait or fake news, or real news.

Assume a production function

$$
F_{i}=A_{i} f_{i}^{k_{i}}
$$


for clickbait or fake news, where $A_{i}>0$ is the production efficiency and $k_{i}$ is a parameter which determines concave production when $0<k_{i}<1$, linear production when $k_{i}=1$, and convex production when $k_{i}>1$. Assume a production function

$$
R_{i}=D_{i} r_{i}^{h_{i}}=D_{i}\left(\frac{b_{i}-a_{i} f_{i}}{d_{i}}\right)^{h_{i}}
$$

for real news, where (1) has been inserted, $D_{i}>0$ is the production efficiency, and $h_{i}$ is a parameter which determines concave production when $0<h_{i}<1$, linear production when $h_{i}=1$, and convex production when $h_{i}>1$.

We measure $F_{i}$ and $R_{i}$ in various ways, such as number of views, advertising revenue, subscriptions, etc. A cheaply produced news item with a catchy heading may potentially be a huge and heavily consumed news item. An expensively produced documentary may be equally much consumed, but is more expensive to produce.

News organisation $i$ cannot be expected to announce its effort $f_{i}$ publicly. Its management deliberately or implicitly chooses $f_{i}$, as observed by neutral fact checkers. We define $f_{i}=0$ as no effort towards clickbait or fake news, i.e., $100 \%$ effort towards real news, and $f_{i}=f_{i}^{\max }=b_{i} / a_{i}$ as $100 \%$ effort towards clickbait or fake news. The benchmark $f_{i}=0$ of no effort towards clickbait or fake news is assumed descriptive of an ideologically neutral and independent news organisation focused on providing real news to the customers. The other extreme $f_{i}=b_{i} / a_{i}$ can describe an ideologically biased news organisation, possibly controlled by an ideologically biased company, and possibly providing news to gullible consumers with limited or no ability or interest to distinguish between clickbait or fake news, and real news.

We assume a fraction $g_{i}$ of gullible consumers towards news organisation $i, 0 \leq g_{i} \leq 1$, consuming both clickbait or fake news, and real news. The clickbait or fake news is often or usually designed and presented to get more attention and thus consumption. We model this so that the fraction $g_{i}$ of gullible consumers on average consumes an item of real news $v_{i}$ times as likely as an item of clickbait or fake news, where $0 \leq v_{i} \leq 1$. Thus,

$$
G_{i}=F_{i}+v_{i} R_{i}
$$

measures news consumption from organisation $i$ by the gullible consumers. That is, $v_{i}=0$ means that only clickbait or fake news is consumed, while $v_{i}=1$ means that all items of news are equally consumed.

Not all consumers are gullible. Otherwise, no upper limit would exist for clickbait or fake news. The remaining fraction $1-g_{i}$ of consumers consume only real news, and no clickbait or fake news from news organisation $i$. These consumers have incorporated their preference for real news into their news consumption. They consume real news 
more conscientiously and deliberately. We assume that they fact check across sources and are able to distinguish real news from clickbait or fake news. We do not model external regulators, fact checkers, watchdogs, and law enforcement impacting players, preferences, and utilities.

News organisation $i$ receives its utility from consumption by the fraction $g_{i}$ of gullible consumers, and the fraction $1-g_{i}$ of non-gullible consumers. If the fraction $g_{i}$ is large, news organisation $i$ prefers to exert high effort $f_{i}$ towards producing clickbait or fake news. In contrast, if $g_{i}$ is low, news organisation $i$ prefers to exert low effort $f_{i}$ to attract the non-gullible consumers.

With these assumptions, and assuming the Cobb-Douglas utility with elasticities $g_{i}$ and $1-g_{i}$ for the gullible and non-gullible consumers, respectively, news organisation $i$ 's utility is

$$
u_{i}=G_{i}^{g_{i}} R_{i}^{1-g_{i}}=\left(A_{i} f_{i}^{k_{i}}+v_{i} D_{i}\left(\frac{b_{i}-a_{i} f_{i}}{d_{i}}\right)^{h_{i}}\right)^{g_{i}}\left(D_{i}\left(\frac{b_{i}-a_{i} f_{i}}{d_{i}}\right)^{h_{i}}\right)^{1-g_{i}}
$$

where (1), (3), (4) and (5) have been inserted. We apply the Cobb-Douglas utility since it is one of the most commonly used utility functions in economics, especially applicable when a balance or trade-off has to be struck between two objectives, such as recruiting gullible versus non-gullible consumers. The Cobb-Douglas utility is often also analytically tractable, at least to some extent, see Appendix. Equation (6) shows how organisation $i$ strikes a balance between choosing high effort $f_{i}$ to attract the gullible consumers, and low effort $f_{i}$ to attract the non-gullible consumers.

Let us consider the two extreme alternatives of (6). First, assume that news organisation $i$ prefers only non-gullible consumers, i.e., ignores its gullible consumers. Inserting $g_{i}=0$ into (6) gives

$$
u_{i}=D_{i}\left(\frac{b_{i}-a_{i} f_{i}}{d_{i}}\right)^{h_{i}}
$$

which is maximised by choosing zero $f_{i}^{*}=0$ into producing clickbait or fake news, and thus maximum effort $r_{i}^{*}=r_{i}^{\max }=b_{i} / d_{i}$ into producing real news, causing utility $u_{i}^{*}=D_{i}\left(b_{i} / d_{i}\right)^{h_{i}}$, where superscript * means optimal value. In order to make this problem more interesting, assume that news organisation $i$ actually has to strike a balance between two objectives, so that $g_{i}$ is intermediate between 0 and 1 . The two types of consumers may be relabelled as extremely non-gullible and moderately non-gullible consumers, or as interested in two different subject areas, such as politics and science. Second, assume that news organisation $i$ prefers only gullible consumers, i.e., ignores its non-gullible consumers. Inserting $g_{i}=1$ into (6) gives 


$$
u_{i}=A_{i} f_{i}^{k_{i}}+v_{i} D_{i}\left(\frac{b_{i}-a_{i} f_{i}}{d_{i}}\right)^{h_{i}}
$$

If $v_{i}>0$, then $f_{i}^{*}$ is intermediate between $f_{i}^{*}=0$ and $f_{i}=f_{i}^{\max }=b_{i} / a_{i}$. However, if $v_{i}=0$, so that the gullible consumers have no added interest in real news, news organisation $i$ maximizes its utility in (8) by choosing maximum effort $f_{i}^{*}=f_{i}^{\text {max }}=b_{i} / a_{i}$ into producing clickbait or fake news, causing utility $u_{i}^{*}=A_{i}\left(b_{i} / a_{i}\right)^{k_{i}}$. Also, for this alternative, in order to make this problem more interesting, assume that news organisation $i$ actually has to strike a balance between two objectives, so that $g_{i}$ is intermediate between 0 and 1 . The two types of consumers may be relabelled as extremely gullible and moderately gullible consumers, or as interested in two different subject areas within which gullibility may play a role, such as politics and entertainment.

We define news organisation $i$ 's trustworthiness $T_{i}$ as its ratio of produced real news $R_{i}$ relative to its produced news if it were to choose effort $f_{i}=0$ towards producing clickbait or fake news, i.e.

$$
T_{i}=\frac{R_{i}}{D_{i}\left(\frac{b_{i}}{d_{i}}\right)^{h_{i}}}=\frac{D_{i}\left(\frac{b_{i}-a_{i} f_{i}}{d_{i}}\right)^{h_{i}}}{D_{i}\left(\frac{b_{i}}{d_{i}}\right)^{h_{i}}}=\frac{\left(b_{i}-a_{i} f_{i}\right)^{h_{i}}}{b_{i}^{h_{i}}}
$$

Hence, no trustworthiness means $T_{i}=0$, and maximum trustworthiness means $T_{i}=1$, where $0 \leq T_{i} \leq 1$.

\subsection{Analysis}

To determine its optimal effort $f_{i}$, news organisation $i$ differentiates its utility in (6) and equates with zero, i.e.

$$
\begin{aligned}
\frac{d u_{i}}{d f_{i}}= & \frac{\left(D_{i}\left(\frac{b_{i}-a_{i} f_{i}}{d_{i}}\right)^{h_{i}}\right)^{1-g_{i}}\left(A_{i} f_{i}^{k_{i}}+v_{i} D_{i}\left(\frac{b_{i}-a_{i} f_{i}}{d_{i}}\right)^{h_{i}}\right)^{-1+g_{i}}}{f_{i}\left(b_{i}-a_{i} f_{i}\right)} \\
& \times\left(A_{i} f_{i}^{k_{i}}\left(\left(b_{i}-a_{i} f_{i}\right) g_{i} k_{i}-a_{i} f_{i}\left(1-g_{i}\right) h_{i}\right)-a_{i} D_{i} f_{i}\left(\frac{b_{i}-a_{i} f_{i}}{d_{i}}\right)^{h_{i}} h_{i} v_{i}\right)=0
\end{aligned}
$$

Setting $k_{i}=h_{i}=1$, solving (10) when $A_{i} d_{i} g_{i} \geq a_{i} D_{i} g_{i}$ gives 


$$
f_{i}^{*}=\frac{b_{i}\left(A_{i} d_{i} g_{i}-a_{i} D_{i} v_{i}\right)}{a_{i}\left(A_{i} d_{i}-a_{i} D_{i} v_{i}\right)}, \quad u_{i}^{*}=\frac{A_{i} b_{i} D_{i}^{1-g_{i}} g_{i}^{g_{i}}\left(1-g_{i}\right)^{1-g_{i}}}{a_{i}^{g_{i}}\left(A_{i} d_{i}-a_{i} D_{i} v_{i}\right)^{1-g_{i}}}
$$

where superscript * means optimal value. When $A_{i} d_{i} g_{i}<a_{i} D_{i} v_{i}$, then $\frac{d u_{i}}{d f_{i}}<0$ for all $f_{i}>0$, and hence

$$
f_{i}^{*}=0, \quad u_{i}^{*}=\frac{b_{i} D_{i} v_{i}^{g_{i}}}{d_{i}}
$$

Differentiating (10) and inserting (11) gives

$$
\left.\frac{d^{2} u_{i}}{d f_{i}^{2}}\right|_{f_{i}=f_{i}^{*}}=-\frac{a_{i}^{2-g_{i}} D_{i}^{1-g_{i}}\left(A_{i} d_{i}-a_{i} D_{i} v_{i}\right)^{1+g_{i}}}{A_{i} b_{i} d_{i}^{2}\left(1-g_{i}\right)^{g_{i}} g_{i}^{1-g_{i}}}
$$

which is negative when $A_{i} d_{i} \geq a_{i} D_{i} v_{i}$, which is satisfied when $A_{i} d_{i} g_{i} \geq a_{i} D_{i} v_{i}$ in (11). Hence, the second order conditions are satisfied when $k_{i}=h_{i}=1$.

Property 1. If $k_{i}=h_{i}=1$ and $A_{i} d_{i} g_{i}>a_{i} D_{i} v_{i}$,

$$
\frac{d f_{i}^{*}}{d b_{i}} \geq 0, \frac{d f_{i}^{*}}{d a_{i}} \leq 0, \frac{d f_{i}^{*}}{d d_{i}} \geq 0, \frac{d f_{i}^{*}}{d A_{i}} \geq 0, \frac{d f_{i}^{*}}{d D_{i}} \leq 0, \frac{d f_{i}^{*}}{d g_{i}} \geq 0, \frac{d f_{i}^{*}}{d v_{i}} \leq 0
$$

if $k_{i}=h_{i}=1$ and $A_{i} d_{i} g_{i} \leq a_{i} D_{i} v_{i}, f_{i}=0$.

Proof. Follows from Appendix and (11).

Property 1 states that for the interior solution where $k_{i}=h_{i}=1$ and $A_{i} d_{i} g_{i}>a_{i} D_{i} v_{i}$, news organisation $i$ 's effort $f_{i}^{*}$ to produce clickbait or fake news increases as the following four parameter values increase. First, its budget $b_{i}$, which enables higher effort. Second, its unit effort cost $d_{i}$ to produce real news, which makes it more expensive to produce real news. Third, its production efficiency $A_{i}$ for clickbait or fake news, which causes effort $f_{i}^{*}$ to have higher impact. Fourth, the fraction $g_{i}$ of consumers consuming clickbait or fake news, which incentivises news organisation $i$ to exert higher effort $f_{i}^{*}$.

In contrast, $f_{i}^{*}$ decreases as the following three parameter values increase. First, its unit effort $\operatorname{cost} a_{i}$ to produce clickbait or fake news, which makes it more costly to exert effort $f_{i}^{*}$. Second, the production efficiency $D_{i}$ for real news, which incentivises exerting effort $r_{i}^{*}$ to produce real news rather than effort $f_{i}^{*}$ to produce clickbait or fake news. Third, how many times as likely, i.e., $v_{i}$, the fraction $g_{i}$ of gullible consumers 
consumes an item of real news rather than an item of clickbait or fake news. The parameter $v_{i}$ places larger emphasis on real news in how gullible consumers consume news, as expressed by $G_{i}$ in (5).

Property 2. If $k_{i}=h_{i}=1$ and $A_{i} d_{i} g_{i} \geq a_{i} D_{i} v_{i}$,

$$
\begin{aligned}
& \frac{d u_{i}^{*}}{d b_{i}} \geq 0, \quad \frac{d u_{i}^{*}}{d a_{i}} \leq 0, \frac{d u_{i}^{*}}{d d_{i}} \leq 0, \quad \frac{d u_{i}^{*}}{d A_{i}} \geq 0, \frac{d u_{i}^{*}}{d D_{i}} \geq 0, \frac{d u_{i}^{*}}{d g_{i}} \leq 0 \\
& \text { if } \frac{a_{i} D_{i}\left(1-g_{i}\right)}{g_{i}\left(A_{i} d_{i}-a_{i} D_{i} v_{i}\right)} \geq 1, \quad \frac{d u_{i}^{*}}{d v_{i}} \geq 0 . \\
& \text { If } k_{i}=h_{i}=1 \text { and } A_{i} d_{i} g_{i}<a_{i} D_{i} v_{i}, \\
& \frac{d u_{i}^{*}}{d b_{i}} \geq 0, \frac{d u_{i}^{*}}{d a_{i}}=0, \frac{d u_{i}^{*}}{d d_{i}} \leq 0, \quad \frac{d u_{i}^{*}}{d A_{i}}=0, \frac{d u_{i}^{*}}{d D_{i}} \geq 0, \frac{d u_{i}^{*}}{d g_{i}} \geq 0 .
\end{aligned}
$$

Proof. Appendix.

Property 2 states that for the interior solution, where $k_{i}=h_{i}=1$ and $A_{i} d_{i} g_{i} \geq a_{i} D_{i} v_{i}$, news organisation $i$ 's utility $u_{i}^{*}$ increases as the following four parameter values increase. First, its budget $b_{i}$, which is beneficial. Second, its production efficiency $A_{i}$ for clickbait or fake news, which causes more clickbait or fake news for a given effort $f_{i}^{*}$. Third, its production efficiency $D_{i}$ for real news, which causes more real news for a given effort $r_{i}^{*}$. Fourth, how many times as likely, i.e., $v_{i}$, the fraction $g_{i}$ of gullible consumers consumes an item of real news rather than an item of clickbait or fake news. A higher parameter value $v_{i}$ gives a higher measure $G_{i}$ in (5) for how gullible consumers consume news, which is beneficial for news organisation $i$.

In contrast, $u_{i}^{*}$ decreases as the following two parameter values increase. First, its unit effort cost $a_{i}$ to produce clickbait or fake news, which causes news organisation $i$ to incur a higher cost for a given effort $f_{i}^{*}$. Second, its unit effort cost $d_{i}$ to produce real news, which causes news organisation $i$ to incur a higher cost for a given effort $r_{i}^{*}$.

Finally, $u_{i}^{*}$ decreases as the fraction $g_{i}$ of consumers consuming clickbait or fake news increases provided that $\frac{a_{i} D_{i}\left(1-g_{i}\right)}{g_{i}\left(A_{i} d_{i}-a_{i} D_{i} v_{i}\right)}>1$, increases as $g_{i}$ increases provided that $\frac{a_{i} D_{i}\left(1-g_{i}\right)}{g_{i}\left(A_{i} d_{i}-a_{i} D_{i} v_{i}\right)}<1$, and is otherwise independent of $g_{i}$. When $g_{i}$ is low while, $A_{i} d_{i} g_{i} \geq a_{i} D_{i} v_{i}, \frac{a_{i} D_{i}\left(1-g_{i}\right)}{g_{i}\left(A_{i} d_{i}-a_{i} D_{i} v_{i}\right)}>1$ is satisfied, causing $u_{i}^{*}$ to decrease as $g_{i}$ increases. 
As $g_{i}$ approaches $g_{i}=1, \frac{a_{i} D_{i}\left(1-g_{i}\right)}{g_{i}\left(A_{i} d_{i}-a_{i} D_{i} v_{i}\right)}>1$ is not satisfied, causing $u_{i}^{*}$ to increase as $g_{i}$ increases. This suggests that some intermediate fraction $g_{i}$ of gullible consumers causes low utility $u_{i}^{*}$, while lower or higher $g_{i}$ causes higher utility. (11) is $v_{i}=0$, which means that the fraction $g_{i}$ of gullible consumers consumes only clickbait or fake news, and no real news. Inserting $v_{i}=0$ into (11) gives

$$
f_{i}^{*}=\frac{b_{i} g_{i}}{a_{i}}, \quad u_{i}^{*}=\frac{b_{i} A_{i}^{g_{i}} D_{i}^{1-g_{i}} g_{i}^{g_{i}}\left(1-g_{i}\right)^{1-g_{i}}}{a_{i}^{g_{i}} d_{i}^{1-g_{i}}}
$$

\section{Two competing news organisations}

\subsection{The model}

This section considers two competing news organisations $i$ and $j$, which can be interpreted as one organisation $i$ competing against the rest of the news industry expressed as $j$, as shown in Fig. 1 .

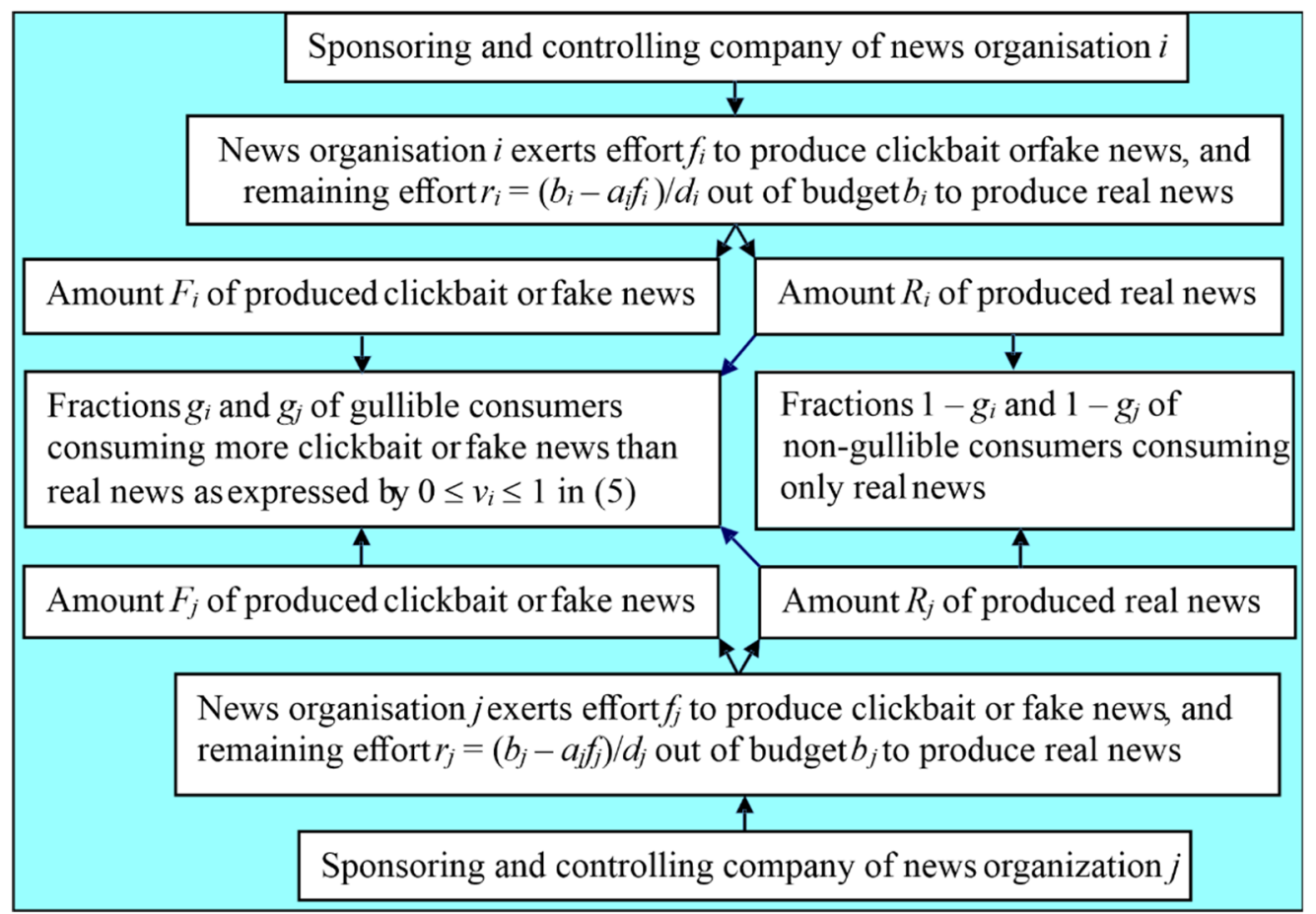

Fig. 1. Two news organisations $i$ and $j$ competing for gullible and non-gullible consumers 
We assume two contests between the two news organisations. The first contest is for the gullible consumers. The second contest is for the non-gullible consumers. The Cobb-Douglas utility elasticities for the two contests are $g_{i}$ and $1-g_{i}$ for organisation $i$, and $g_{j}$ and $1-g_{j}$ for organisation $j$. The two organisations' utilities are

$$
u_{i}=\left(\frac{G_{i}}{G_{i}+G_{j}}\right)^{g_{i}}\left(\frac{R_{i}}{R_{i}+R_{j}}\right)^{1-g_{i}}, u_{j}=\left(\frac{G_{j}}{G_{i}+G_{j}}\right)^{g_{j}}\left(\frac{R_{j}}{R_{i}+R_{j}}\right)^{1-g_{j}}
$$

where $G_{i}$ and $R_{i}$ are given by (5) and (4), and $G_{j}$ and $R_{j}$ are given by (5) and (4) replacing $i$ with $j$. In (15), organisation $i$ seeks high $G_{i}$ relative to $G_{i}$, and high $R_{i}$ relative to $R_{j}$, regulated by $g_{i}$ and $1-g_{i}$, which means that two kinds of balances have to be struck. Organisation $i$ strikes a balance between producing fake news to attract gullible customers and producing real news to attract non-gullible customers, while competing with the other news organisation $j$. More specifically, first, organisation $i$ strikes a balance between choosing high effort $f_{i}$ to attract the gullible consumers in the first ratio in (15), and low effort $f_{i}$ to attract the non-gullible consumers in the second ratio in (15). Second, organisation $i$ strikes a balance between choosing high effort $f_{i}$ to compete with organisation $j$, which also strikes a balance between choosing high effort $f_{i}$ in the first ratio in (15), while simultaneously choosing low effort $f_{i}$ to compete with organisation $j$ also choosing low effort $f_{j}$ in the second ratio in (15). Organisation $j$ reasons analogously. The two news organisations $i$ and $j$ choose their free choice variables $f_{i}$ and $f_{j}$ simultaneously and independently.

\subsection{Analysis}

To determine news organisations $i$ 's and $j$ 's optimal efforts $f_{i}^{*}$ and $f_{j}^{*}$, their utilities in (15) are differentiated and equated to zero, i.e.

$$
\frac{d u_{i}}{d f_{i}}=0, \frac{d u_{j}}{d f_{j}}=0
$$

which for $k_{i}=h_{i}=1$ are solved to yield

$$
f_{i}^{*}=\left\{\begin{array}{l}
\frac{b_{i}\left(\frac{A_{j} d_{j} f_{j}^{*} Q}{b_{j}-a_{j} f_{j}^{*}}-\left(D_{i} v_{i}-D_{j} Q v_{j}\right)\right)}{A_{i} d_{i}+a_{i}\left(\frac{A_{j} d_{j} f_{j}^{*} Q}{b_{j}-a_{j} f_{j}^{*}}\right)-\left(D_{i} v_{i}-D_{j} Q v_{j}\right)} \\
0 \text { otherwise }
\end{array} f_{i i}^{*} \text { if } f_{i i}^{*} \geq 0\right.
$$




$$
Q=\left\{\begin{array}{l}
\sqrt{\frac{\left(a_{j} D_{i} g_{i}\left(1-g_{j}\right)\left(A_{i} d_{i}-a_{i} D_{i} v_{i}\right)\right)}{\left(a_{i} D_{j} g_{j}\left(1-g_{j}\right)\left(A_{j} d_{j}-a_{j} D_{j} v_{j}\right)\right)}} \text { if } A_{i} d_{i} \geq a_{i} D_{i} v_{i} \text { and } \mathrm{A}_{j} d_{j} \geq a_{j} D_{j} v_{j} \\
0 \text { otherwise }
\end{array}\right.
$$

Inserting (17) into $\frac{d u_{j}}{d f_{j}}=0$ in (16) gives a second order equation in $f_{j}^{*}$, which takes too much space to present. For the special case of two equivalent news organisations, i.e., $b_{j}=b_{i}, a_{j}=a_{i}, d_{j}=d_{i}, A_{j}=A_{i}, D_{j}=D_{i}, g_{j}=g_{i}, v_{j}=v_{i}$, (17) simplifies to

$$
f_{i}^{*}=f_{j}^{*}=\left\{\begin{array}{l}
\frac{b_{i}\left(A_{i} d_{i} g_{i}-a_{i} D_{i} v_{i}\right)}{a_{i}\left(A_{i} d_{i}-a_{i} D_{i} v_{i}\right)} \text { if } A_{i} d_{i} g_{i} \geq a_{i} D_{i} v_{i}, u_{i}^{*}=u_{j}^{*}=\frac{1}{2} \\
0 \text { otherwise }
\end{array}\right.
$$

Equation (18) shows the same efforts $f_{i}^{*}=f_{j}^{*}$ as for one news organisation in (11), while the utilities $u_{i}^{*}=u_{j}^{*}$ are always equal for equal efforts because of the contests in (15), which contrasts with no contest for one news organisation.

Figure 2 plots the two news organisations $i$ 's and $j$ 's efforts $f_{i}^{*}, f_{j}^{*}, r_{i}^{*}, r_{j}^{*}$ and utilities $u_{i}^{*}$ and $u_{j}^{*}$ as functions of the seven parameters $b_{i}, a_{i}, d_{i}, A_{i}, D_{i}, g_{i}, v_{i}$ relative to the benchmark $b_{i}=b_{j}=a_{i}=a_{j}=d_{i}=d_{j}=A_{i}=A_{j}=D_{i}=D_{j}=1, g_{i}=g_{j}=1 / 2$, and $v_{i}=v_{j}=1 / 4$. This benchmark is chosen since it is simple, intermediate, and plausible. $g_{i}=g_{j}=1 / 2$ in (6) is intermediate between $g_{i}=g_{j}=0$ and $g_{i}=g_{j}=1$, which means that clickbait or fake news, and real news, have equal elasticities, and thus have equal impact on the utilities $u_{i}^{*}$ and $u_{j}^{*} \cdot v_{i}=v_{j}=1 / 4$ in (5) means that clickbait or fake news is weighted four times more than real news, which may be a plausible assessment of gullible consumers' news consumption. Inserting the benchmark into (18) in all the seven panels the benchmark causes equal efforts $f_{i}^{*}=f_{j}^{*}=1 / 3$ and equal utilities $u_{i}^{*}=u_{j}^{*}=1 / 2$.

In panel a), organisation $i$ 's effort $f_{i}^{*}$ to produce clickbait or fake news, and effort $r_{i}^{*}$ to produce real news increase linearly, and organisation $i$ 's utility $u_{i}^{*}$ increases concavely as its budget $b_{i}$ increases. Increasing budget $b_{i}$ is beneficial for organisation $i$ and causes convexly decreasing utility $u_{j}^{*}$ for organisation $j$ due to lower budget $b_{j}=1$.

In panel b), organisation $i$ 's effort $f_{i}^{*}$ and utility $u_{i}^{*}$ decrease convexly as its unit cost of effort $a_{i}$ to produce clickbait or fake news increases, reaching zero and a constant, respectively, when $a_{i} \geq 2.33$. Too high $a_{i}$ induces organisation $i$ not to produce clickbait or fake news, and instead focus exclusively on producing real news. On the other hand, 

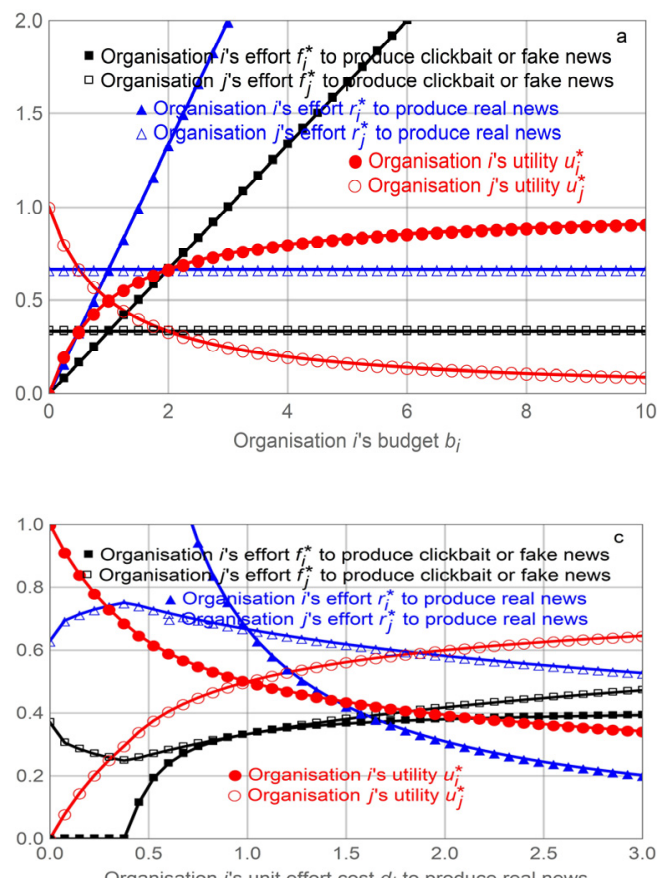

Organisation $i$ 's unit effort cost $d_{j}$ to produce real news

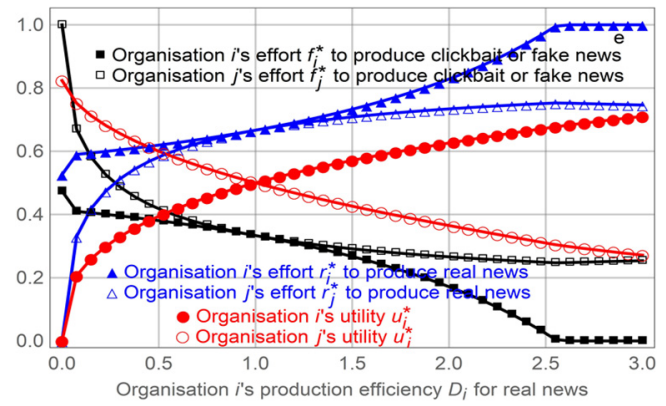

Fig. 2. News organisations $i$ 's and $j$ 's efforts $f_{i}^{*}, f_{j}^{*}, r_{i}^{*}, r_{j}^{*}$ and utilities $u_{i}^{*}$ and $u_{j}^{*}$ as functions of $b_{i}, a_{i}, d_{i}, A_{i}, D_{i}, g_{i}, v_{i}$ relative to the benchmark $b_{i}=b_{j}=a_{i}=a_{j}=d_{i}=d_{j}=A_{i}=A_{j}=D_{i}=D_{j}=1$, $g_{i}=g_{j}=1 / 2$, and $v_{i}=v_{j}=1 / 4$
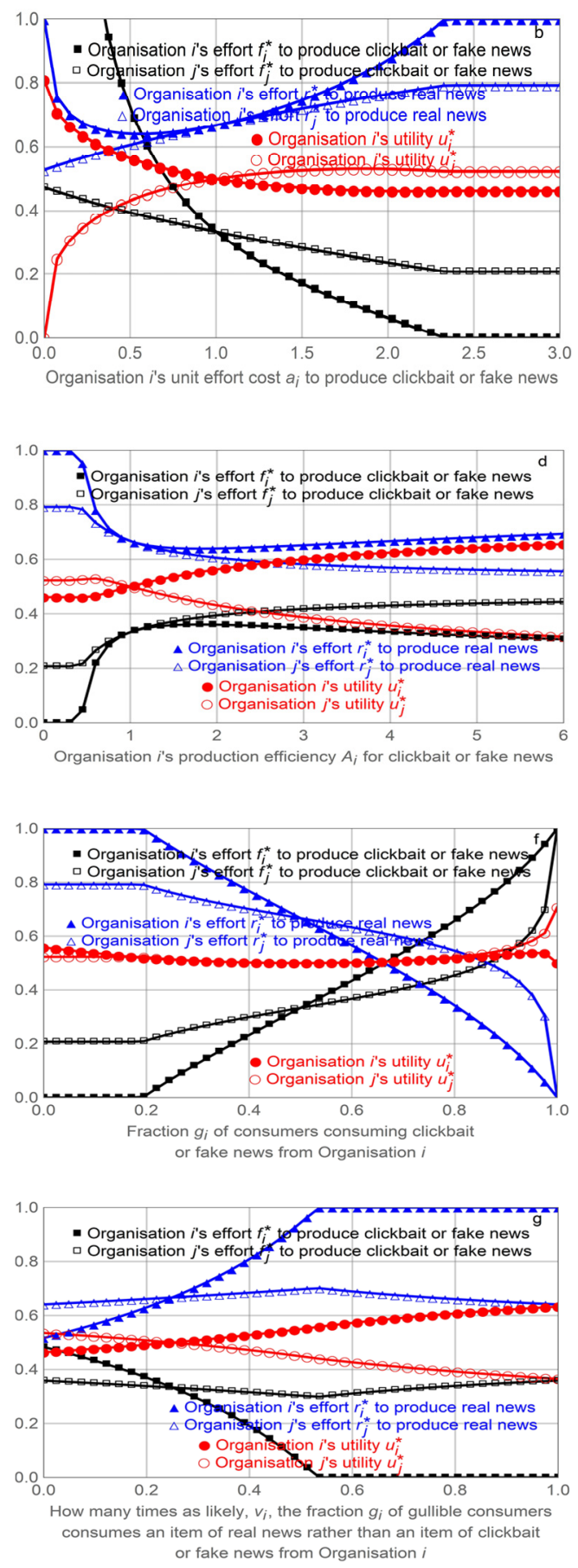
organisation $j$ benefits from increasing $a_{i}$ which enables it to exert lower effort $f_{j}^{*}$ at fixed unit cost $a_{j}=1$, and earn concavely increasing utility $u_{j}^{*}$ which, eventually, decreases slightly towards a constant.

In panel c), organisation $i$ 's effort $f_{i}^{*}$ increases concavely as its unit effort cost $d_{i}$ to produce real news increases, equalling zero when $d_{i} \leq 0.39$. Both too low $d_{i}$, and too high $a_{i}$ induce organisation $i$ not to produce clickbait or fake news, and instead focus exclusively on producing real news. As $d_{i}$ increases, organisation $i$ 's utility $u_{i}^{*}$ decrease convexly, and organisation $j$ 's utility $u_{j}^{*}$ increase concavely. Determined numerically, $\lim _{\substack{d_{i} \rightarrow \infty \\ f_{i}}}=0.41$ and $\lim u_{i}^{*}=0$, since organisation $i$ cannot compete effectively on real news with organi$d_{i} \rightarrow \infty$

sation $j$ when its unit effort cost $d_{i}$ is too high. Furthermore, $\lim f_{j}^{*}=1$, since clickbait or fake news is where the competition is, and $\lim u_{j}^{*}=0.84$.

In panel $\mathrm{d}$ ), organisation $i$ 's effort $f_{i}^{*}$ increases logistically towards a maximum $f_{i}^{*}=0.36$ when $A_{i}=1.80$, and thereafter decreases convexly as its production efficiency $A_{i}$ for clickbait or fake news increases, equalling zero when $A_{i} \leq 0.43$. Too low $A_{i}$ or $d_{i}$, or too high $a_{i}$, causes $f_{i}^{*}=0$ since clickbait or fake news is not cost efficient to produce. Too high $A_{i}$ expresses a luxury problem for organisation $i$ where excessive clickbait or fake news can be produced at low effort $f_{i}^{*}$. Determined numerically, $\lim _{A_{i} \rightarrow \infty} f_{i}^{*}=\lim _{A_{i} \rightarrow \infty} u_{j}^{*}$ $=0, \lim _{A_{i} \rightarrow \infty} u_{i}^{*}=0.81, \lim _{A_{i} \rightarrow \infty} f_{j}^{*}=0.47$.

In panel e), organisation $i$ 's effort $f_{i}^{*}$ decreases convexly (except when $D_{i}$ is very low) as its production efficiency $D_{i}$ for real news increases, reaching zero when $D_{i} \geq 2.56$. Too high $D_{i}$ or $a_{i}$ or too low $A_{i}$ or $d_{i}$ causes $f_{i}^{*}=0$ since clickbait or fake news is not cost efficient to produce. Organisation $i$ benefits from increasing $D_{i}$ which causes its utility to increase concavely, eventually reaching $\lim u_{i}^{*}=1$, where $\lim u_{j}^{*}=0$, and $\lim f_{i}^{*}=0.33$.

$$
D_{i} \rightarrow \infty \quad D_{i} \rightarrow \infty \text {, } D_{i} \rightarrow \infty
$$

In panel $\mathrm{f}$ ), organisation $i$ 's effort $f_{i}^{*}$ increases circa linearly, and eventually slightly convexly, as its fraction $g_{i}$ of consumers consuming clickbait or fake news increases to $g_{i}=1$, equalling zero when $g_{i} \leq 0.19$. Too low elasticity or weight $g_{i} \leq 0.19$ assigned to the contest over clickbait or fake news induces organisation $i$ to focus exclusively on producing real news. Organisation $i$ always produces real news when $g_{i}<1$, which follows from the asymmetry caused by the benchmark where 
$v_{i}=v_{j}=1 / 4$ in $G_{i}=F_{i}+v_{i} R_{i}$ in [6] causes the production of real news to impact the first contest in (15).

In panel g), organisation $i$ 's effort $f_{i}^{*}$ decreases convexly as $v_{i}$ increases, reaching zero when $v_{i} \geq 0,53$, where $v_{i}$ expresses how many times as likely the fraction $g_{i}$ of gullible consumers consumes an item of real news rather than an item of clickbait or fake news from news organisation $i$. Too high $v_{i}$ or $D_{i}$ or $a_{i}$, or too low $A_{i}$ or $d_{i}$, causes $f_{i}^{*}=0$, since clickbait or fake news is not cost efficient to produce. Organisation $i$ benefits from increasing $v_{i}$ which causes increasing utility $u_{i}^{*}$. In contrast, organisation $j$ 's utility $u_{j}^{*}$ decreases as $v_{i}$ increases.

\section{Conclusion}

The article first presents a decision theoretic model, and thereafter a game theoretic model, where two news organisations allocate their budgets between producing clickbait or fake news, and real news. They seek to attract gullible consumers who consume clickbait or fake news more than real news, and non-gullible consumers who consume only real news. The two news organisations compete with each other in one contest over clickbait or fake news, and one contest over real news.

The decision theoretic model shows that increasing a news organisation's budget causes both more clickbait or fake news, and more real news, to be produced. More clickbait or fake news is produced if the news organisation's unit effort cost to produce real news increases, its production efficiency for clickbait or fake news increases, and the fraction of consumers consuming clickbait or fake news increases. In contrast, less clickbait or fake news is produced if a news organisation's unit effort cost to produce clickbait or fake news increases, its production efficiency for real news increases, and the gullible consumers consume real news with a higher frequency. The news organisation's utility increases if its budget increases, its production efficiencies for clickbait or fake news, and real news, increase, and if the gullible consumers consume more real news in addition to clickbait or fake news. In contrast, the news organisation's utility decreases if its unit effort costs to produce clickbait or fake news, and real news, increase.

The game theoretic model illustrates seven insights. First, higher budget for one news organisation causes higher effort and utility for this news organisation, and constant effort and decreasing utility for the other news organisation.

Second, higher unit effort cost for clickbait or fake news causes lower and, eventually, no effort, and lower utility. The competing news organisation receives overall higher utility. 
Third, low unit effort cost for real news causes no effort to produce clickbait or fake news, and high utility. Higher unit effort cost for real news causes higher effort to produce clickbait or fake news, and lower utility. The competing news organisation receives overall higher utility.

Fourth, sufficiently low production efficiency for clickbait or fake news causes no effort to produce clickbait or fake news. Higher production efficiency for clickbait or fake news causes inverse U-shaped effort to produce clickbait or fake news. For intermediate production efficiency, the competition with the other news organisation is fierce and their utilities are similar. As the production efficiency increases above intermediate, the competition becomes less fierce, and the news organisation can cut back on its effort while receiving higher utility. The competing news organisation receives lower utility.

Fifth, higher production efficiency for real news causes lower effort to produce clickbait or fake news, and higher utility. The competing news organisation receives lower utility. Sufficiently high production efficiency for real news causes no effort to produce clickbait or fake news.

Sixth, sufficiently low weight assigned to the contest over clickbait or fake news induces the news organisation to exert no effort to produce clickbait or fake news. Higher weight assigned to the contest over clickbait or fake news induces both news organisations to exert higher effort to produce clickbait or fake news.

Seventh, as the gullible consumers become more likely to consume real news in addition to fake news, the news organisation exerts lower, and eventually no effort, to produce clickbait or fake news, while receiving higher utility. The competing news organisation receives lower utility.

Future research should incorporate more characteristics into the model, generalise to more than two news organisations, account for dynamics through time, and compile empirical support.

Future research may also assess whether owner biases are more or less prominent than market biases. For example, consumers may reinforce their biases by consuming biased news which may reflect confirmation bias. This, in turn, may reflect social dynamics, such as pluralistic ignorance and group identity, and whether a consumer is a self-respecting member of a politically aligned or biased group. Such consumers may express consternation or outrage at the bias in the media organisations aligned against one's own group, and satisfaction at the bias aligned with one's own group, to minimise internal cognitive dissonance.

Future research may also consider alignment of fake content with biased narratives, and the malinformation problem where a package of facts is selectively pruned to support a biased narrative. This is a common scenario where the effort to produce fake news is comparable or equal to the effort to produce real news. Empirically, this phenomenon is frequently observed, and may represent an equilibrium between competing media producers. 


\section{Nomenclature}

\section{Parameters}

$b_{z}-$ news organisation $z$ 's budget, $z=i, j$

$a_{z}-$ news organisation $z$ 's unit effort cost to produce clickbait or fake news

$d_{z}-$ news organisation $z$ 's unit effort cost to produce real news

$A_{z}-$ news organisation $z$ 's production efficiency for clickbait or fake news

$D_{z}-$ news organisation $z$ 's production efficiency for real news

$k_{z}$ - news organisation $z$ 's parameter for nonlinear impact in production function for clickbait or fake news

$h_{z}$ - news organisation $z$ 's parameter for nonlinear impact in production function for real news

$g_{z}$ - fraction of consumers consuming clickbait or fake news from news organisation $z, 0 \leq g_{z} \leq 1$

$v_{z}$ - how many times as likely the fraction $g_{z}$ of gullible consumers consumes an item of real news rather than an item of clickbait or fake news from news organisation $z, 0 \leq v_{z} \leq 1$

\section{Independent variable}

$f_{z}-$ news organisation $z$ 's effort to produce clickbait or fake news

\section{Dependent variables}

$r_{z}-$ news organisation $z$ 's effort to produce real news

$F_{z}-$ news organisation $z$ 's production function for clickbait or fake news

$R_{z}$ - news organisation $z$ 's production function for real news

$G_{z}-$ news consumption from organisation $z$ by the gullible consumers

$T_{z}-$ trustworthiness of news organisation $z$

$u_{z}-$ news organisation $z$ 's utility

\section{Acknowledgement}

I thank Jun Zhuang for suggesting an analysis of clickbait or fake news, and useful discussions in the early development of the article. I thank two anonymous referees of this journal for useful comments.

\section{References}

[1] Allcott H., GentZkow M., Social Media and Fake News in the 2016 Election, J. Econ. Perspect., 2017, $31(2), 211-236$.

[2] BlOm J.N., HANSEN K.R., Click Bait: Forward-Reference as Lure in Online News Headlines, J. Pragm., 2015, 76, 87-100.

[3] Hausken K., A Cost-Benefit Analysis of Terrorist Attacks, Def. Peace Econ., 2018, 29 (2), 111-129.

[4] Hausken K., A Game Theoretic Model of Adversaries and Media Manipulation, Games, 2019, 10 (4), 48, https://doi.org/10.3390/g10040048

[5] Khoja N., 7 Reasons Why Clicking This Title Will Prove Why You Clicked This Title, 2016, https://venngage. $\mathrm{com} / \mathrm{blog} / 7$-reasons-why-clicking-this-title-will-prove-why-you-clicked-this-title/, accessed July 4, 2020.

[6] Kshetri N., VOAS J., The Economics of “Fake News”, IT Prof., 2017, 19 (6), 8-12.

[7] Levins R., The Strategy of Model Building in Population Biology, Am. Sci., 1966, 54 (4), 421-431. [online]. Available: http://www.jstor.org/stable/27836590 
[8] LuTz A., These 6 Corporations Control 90\% of The Media in America, 2012. [Online]. Available: $\mathrm{https}: / / \mathrm{www}$. businessinsider.com/these-6-corporations-control-90-of-the-media-in-america-2012-6? $\mathrm{r}=\mathrm{US}$ $\& \mathrm{IR}=\mathrm{T} \& \mathrm{IR}=\mathrm{T}$

[9] Nagy L., Fairbrother A., Etterson M., Orme-Zavaleta J., The Intersection of Independent Lies: Increasing Realism in Ecological Risk Assessment, Human Ecol. Risk Assess., Int. J., 2007, 13 (2), 355-369, DOI: 10.1080/10807030701226814.

[10] Subramanian S., Inside the Macedonian Fake-News Complex, 2017, https://www.wired.com/2017/02/ veles-macedonia-fake-news/, accessed July 4, 2020.

[11] TriBe L.H., Policy Science: Analysis or Ideology?, Phil. Publ. Affairs, 1972, 2 (1), 66-110.

[12] Zannettou S., Chatzis S., Papadamou K., Sirivianos M., The Good, the Bad and the Bait: Detecting and Characterizing Clickbait on YouTube, 2018 IEEE Security and Privacy Workshops (SPW), San Francisco, CA, USA. [Online]. Available: https://ieeexplore.ieee.org/document/8424634/authors \#authors

\section{Appendix. Properties 1 and 2}

Differentiating $f_{i}$ in (11) with respect to the various parameters when $A_{i} d_{i} g_{i}>a_{i} D_{i} v_{i}$ gives

$$
\begin{aligned}
& \frac{d f_{i}^{*}}{d b_{i}}=\frac{A_{i} d_{i} g_{i}-a_{i} D_{i} v_{i}}{a_{i}\left(A_{i} d_{i}-a_{i} D_{i} v_{i}\right)} \geq 0, \quad \frac{d f_{i}^{*}}{d a_{i}}=-\frac{b_{i} g_{i}}{a_{i}^{2}}-\frac{b_{i} D_{i}^{2} v_{i}^{2}\left(1-g_{i}\right)}{\left(A_{i} d_{i}-a_{i} D_{i} v_{i}\right)^{2}} \leq 0 \\
& \frac{d f_{i}^{*}}{d d_{i}}=\frac{b_{i} A_{i} D_{i} v_{i}\left(1-g_{i}\right)}{\left(A_{i} d_{i}-a_{i} D_{i} v_{i}\right)^{2}} \geq 0, \quad \frac{d f_{i}^{*}}{d A_{i}}=\frac{b_{i} d_{i} D_{i} v_{i}\left(1-g_{i}\right)}{\left(A_{i} d_{i}-a_{i} D_{i} v_{i}\right)^{2}} \geq 0 \\
& \frac{d f_{i}^{*}}{d D_{i}}=\frac{b_{i} d_{i} A_{i} v_{i}\left(1-g_{i}\right)}{\left(A_{i} d_{i}-a_{i} D_{i} v_{i}\right)^{2}} \leq 0, \quad \frac{d f_{i}^{*}}{d g_{i}}=\frac{b_{i} A_{i} d_{i}}{a_{i}\left(A_{i} d_{i}-a_{i} D_{i} v_{i}\right)} \geq 0 \\
& \frac{d f_{i}^{*}}{d v_{i}}=-\frac{b_{i} d_{i} A_{i} D_{i}\left(1-g_{i}\right)}{\left(A_{i} d_{i}-a_{i} D_{i} v_{i}\right)^{2}} \leq 0
\end{aligned}
$$

Differentiating $u_{i}$ in (11) and (12) with respect to the various parameters gives

$$
\begin{aligned}
& \frac{d u_{i}^{*}}{d b_{i}}=\left\{\begin{array}{l}
\frac{A_{i} D_{i}^{1-g_{i}} g_{i}^{g_{i}}\left(1-g_{i}\right)^{1-g_{i}}}{a_{i}^{g_{i}}\left(A_{i} d_{i}-a_{i} D_{i} v_{i}\right)^{1-g_{i}}} \geq 0 \text { if } A_{i} d_{i} g_{i} \geq a_{i} D_{i} v_{i} \\
\frac{D_{i} v_{i}^{g_{i}}}{d_{i}} \geq 0 \text { otherwise }
\end{array}\right. \\
& \frac{d u_{i}^{*}}{d a_{i}}=\left\{\begin{array}{l}
-\frac{A_{i} b_{i} D_{i}^{1-g_{i}} g_{i}^{g_{i}}\left(1-g_{i}\right)^{1-g_{i}}\left(A_{i} d_{i} g_{i}-a_{i} D_{i} v_{i}\right)}{a_{i}^{1+g_{i}}\left(A_{i} d_{i}-a_{i} D_{i} v_{i}\right)^{2-g_{i}}} \leq 0 \text { if } A_{i} d_{i} g_{i} \geq a_{i} D_{i} v_{i} \\
0 \text { otherwise }
\end{array}\right.
\end{aligned}
$$


$\frac{d u_{i}^{*}}{d d_{i}}=\left\{\begin{array}{l}-\frac{A_{i}^{2} b_{i} D_{i}^{1-g_{i}} g_{i}^{g_{i}}\left(1-g_{i}\right)^{2-g_{i}}}{a_{i}^{g_{i}}\left(A_{i} d_{i}-a_{i} D_{i} v_{i}\right)^{2-g_{i}}} \leq 0 \text { if } A_{i} d_{i} g_{i} \geq a_{i} D_{i} v_{i} \\ -\frac{b_{i} D_{i} v_{i}^{g_{i}}}{d_{i}^{2}} \leq 0 \text { otherwise }\end{array}\right.$
$\frac{d u_{i}^{*}}{d A_{i}}=\left\{\begin{array}{l}\frac{b_{i} D_{i}^{1-g_{i}} g_{i}^{g_{i}}\left(1-g_{i}\right)^{1-g_{i}}\left(A_{i} d_{i} g_{i}-a_{i} D_{i} v_{i}\right)}{a_{i}^{g_{i}}\left(A_{i} d_{i}-a_{i} D_{i} v_{i}\right)^{2-g_{i}}} \geq 0 \text { if } A_{i} d_{i} g_{i} \geq a_{i} D_{i} v_{i} \\ 0 \text { otherwise }\end{array}\right.$

$\frac{d u_{i}^{*}}{d D_{i}}=\left\{\begin{array}{l}\frac{A_{i}^{2} b_{i} d_{i} g_{i}^{g_{i}}\left(1-g_{i}\right)^{2-g_{i}}}{a_{i}^{g_{i}} D_{i}^{g_{i}}\left(A_{i} d_{i}-a_{i} D_{i} v_{i}\right)^{2-g_{i}}} \geq 0 \text { if } A_{i} d_{i} g_{i} \geq a_{i} D_{i} v_{i} \\ \frac{b_{i} v_{i}^{g_{i}}}{d_{i}} \geq 0 \text { otherwise }\end{array}\right.$

$\frac{d u_{i}^{*}}{d g_{i}}=\left\{\begin{array}{l}-\frac{b_{i} A_{i} D_{i}^{1-g_{i}} g_{i}^{g_{i}}\left(1-g_{i}\right)^{1-g_{i}}}{a_{i}^{g_{i}}\left(A_{i} d_{i}-a_{i} D_{i} v_{i}\right)^{1-g_{i}}} \ln \left(\frac{a_{i} D_{i}\left(1-g_{i}\right)}{g_{i}\left(A_{i} d_{i}-a_{i} D_{i} v_{i}\right)}\right) \leq 0 \text { if } A_{i} d_{i} g_{i} \geq a_{i} D_{i} v_{i} \\ \frac{b_{i} D_{i} v_{i}^{g_{i}} \ln v_{i}}{d_{i}} \leq 0 \text { otherwise }\end{array}\right.$ $\frac{d u_{i}^{*}}{d v_{i}}=\left\{\begin{array}{l}\frac{b_{i} A_{i} a_{i}^{1-g_{i}} D_{i}^{2-g_{i}} g_{i}^{g_{i}}\left(1-g_{i}\right)^{2-g_{i}}}{\left(A_{i} d_{i}-a_{i} D_{i} v_{i}\right)^{2-g_{i}}} \geq 0 \text { if } A_{i} d_{i} g_{i} \geq a_{i} D_{i} v_{i} \\ \frac{b_{i} D_{i} g_{i}}{d_{i} v_{i}^{1-g_{i}}} \geq 0 \text { otherwise }\end{array}\right.$ 